\title{
The Synthesis and Evaluation of Hydrophobic Association Thickener
}

\author{
Qingwang Liu, Dong Cheng, Jigang Wang, Zhenzhong Fan, Tong Zhang, Ao Sun \\ EOR Key Laboratory of the Ministry of Education, Northeast Petroleum University, Daqing, China \\ Email: LIUQINGWANG@163.com, orangecd@163.com, wangjigang9999@163.com, fanzhenzhong@163.com, \\ 648553814@qq.com, sunsunaoao@126.com
}

Received 23 October 2014; revised 25 November 2014; accepted 5 December 2014

Copyright @ 2015 by authors and Scientific Research Publishing Inc.

This work is licensed under the Creative Commons Attribution International License (CC BY). http://creativecommons.org/licenses/by/4.0/

(c) (i) Open Access

\section{Abstract}

The paper provides a new hydrophobic association polymer fracturing fluid gelatinizer. The hydrophobic association which is the synthesis of three component copolymer complexes, is compounded by micellar copolymerization method. The hydrophobic association is got together through hydrophilic monomer acrylamide monomer $\mathrm{N}, \mathrm{N}$-vinyl pyrrolidone monomer with a rigid group $\mathrm{N}$, and hydrophobic monomer $\mathrm{N}, \mathrm{N}-2$ dodecyl acrylamide etc. three monomers with sodium dodecyl sulfate as the emulsifier in micellar solution.

\section{Keywords}

Hydrophobic Association, Fracturing Fluid, Surface Active Agent, Three Component Copolymer, Apparent Viscosity

\section{Introduction}

With the development of oil and gas in oil field, most of the oil fields have already entered the late stage of exploitation in our country. Low permeability reservoir has been developed in pursuit of production. Oilfield faces that the formation permeability is low, the quantity of produced fluid in oil wells is little, the injection pressure in water wells is large, etc. Hydraulic fracturing is a very effective stimulation method for oil and water wells. The performance of fracturing fluid is directly related to the quality of the fracturing. Special performance of fracturing fluid is mainly on the performance of the fracturing fluid thickener.

The hydrophobic association synthesized by micellar copolymerization method is embedded hydrophobic association, which has the hydrophobic effect. Vinyl pyrrolidone with a rigid group $\mathrm{N}$ has steric-hinerance effect, which can improve the heat resistance and salt resistance of associated content. N,N-2 dodecyl acrylamide is a twin tail hydrophobic monomer which has more hydrophobic density and association ability. The components of 
hydrophobic groups do not change with the change of the percent conversion in the process of polymerization reaction [1]-[5].

The hydrophobic association has hydrophobic groups, which can occur in water solution. When concentration is higher than the critical concentration of hydrophobic association $\left(C^{*}\right)$, intermolecular association can happen. The association is complex to form a three-dimensional network structure, form a super large molecule, and affect the rheological properties of solution. The association is a physical effect and reversible, which means mesh structure can give recovery after shear failure, and it has shear thinning, shear resistance and shear resistance resilience.

\section{Experimental and Results}

\subsection{The Synthesis of Hydrophobic Association Thickener}

Use Micellar copolymerization method to synthesis hydrophobic association thickener, make the mass fraction of $3 \%$ sodium dodecyl sulfate micellar solution. In three monomer molar ratios of acrylamide: $N$-vinyl pyrrolidone:N,N-2 dodecyl acrylamide $=80: 20: 1.2$ dissolved in micellar solution. Total monomer mass concentration of $20 \%$. Stir to dissolve, so that the acrylamide and N-vinyl pyrrolidone soluble in water, N,N-2 dodecyl acrylamide dissolved in micelle. Pour the configured micellar solution into a flask with mixer, control reaction temperature $40^{\circ} \mathrm{C}$ in constant temperature water bath, keep stirring.

To the solution into the nitrogen after 30 minutes, add solution in the solution of $0.1 \%$ of the total mass quality ammonium sulfate initiator, seal reaction container in 10 minutes after keeping nitrogen. Keep action $4 \mathrm{~h}$, get transparent sticky glue, the crude product, wash the crude product with acetone over and over again, remove unreacted monomer and surfactant, cut up the washed products. Baking in the oven at $70^{\circ} \mathrm{C}$, crush into small particles, is the hydrophobic association thickener, value $k, n$ of Hydrophobic association thickener.

\subsection{The Rheological Properties of Thickener}

Rheological parameters are important parameters of the design of fracturing, and have a direct impact to cracks size calculation. At $30^{\circ} \mathrm{C}-70^{\circ} \mathrm{C}$, make $0.8 \%$ thickener solution, use rotational viscometer to test its rheological property.

The experimental results in Table 1 show that the thickening agent has strong characteristics of non-newtonian fluid, good rheological property and carrying capacity.

\subsection{The Shear Stability of the Thickener}

Figure 1 shows viscosity of the hydrophobic association solution shows to be shear thinning behavio, viscosity is decreased with the increase of shear rate; Under the same shear rate, with the loss of the hydrophobic association thickener solution concentration, solution viscosity decreased; When the hydrophobic association after solution concentration is greater than $1.70 \mathrm{~g} / \mathrm{L}$, the shear rate is less than $1 \mathrm{~s}^{-1}$, with the increase of shear rate, the solution viscosity drops rapidly, when the shear rate in $1 \mathrm{~s}^{-1}-10 \mathrm{~s}^{-1}$, solution viscosity with the increase of shear rate, slow down, when the shear rate is more than $10 \mathrm{~s}^{-1}$, viscosity drops rapidly along with the increase of shear rate.

\subsection{Hydrophobic Associating Thickening Performance}

Prepare different concentration thickener, test the viscosity in $50^{\circ} \mathrm{C}$ water bath, $170 \mathrm{~s}^{-1}$ shear rate.

When the concentration of hydrophobic association thickener is not more than $1.7 \mathrm{~g} / \mathrm{L}$, viscosity rise gently, viscosity increasing quantity is limited. When the hydrophobic association solution concentration is greater than $1.7 \mathrm{~g} / \mathrm{L}$, viscosity suddenly rise significantly (Figure 2).

\subsection{The Influence of Temperature on the Hydrophobic Association}

Figure 3 is association of the aqueous solution viscosity-temperature diagram, the viscosity with the increase of temperature decreases, while rigid ring of the association have resistance to temperature effect, but also just to maintain the stability of the macromolecular chain. Temperature caused by hydration of enhancement is the 
Table 1. The rheological properties of thickener.

\begin{tabular}{cccccr}
\hline Temperature $\left({ }^{\circ} \mathrm{C}\right)$ & 30 & 40 & 50 & 60 & 70 \\
\hline $\mathrm{n}$ & 0.501 & 0.498 & 0.484 & 0.482 & 0.476 \\
$\mathrm{~K}(\mathrm{~Pa} . \mathrm{Sn})$ & 0.657 & 0.654 & 0.664 & 0.665 \\
\hline
\end{tabular}

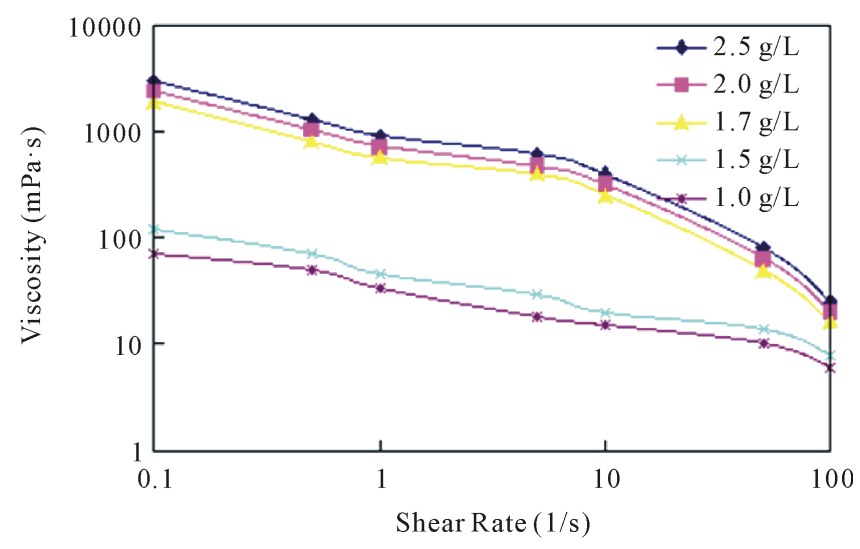

Figure 1. The influence of shear rate on viscosity of association.

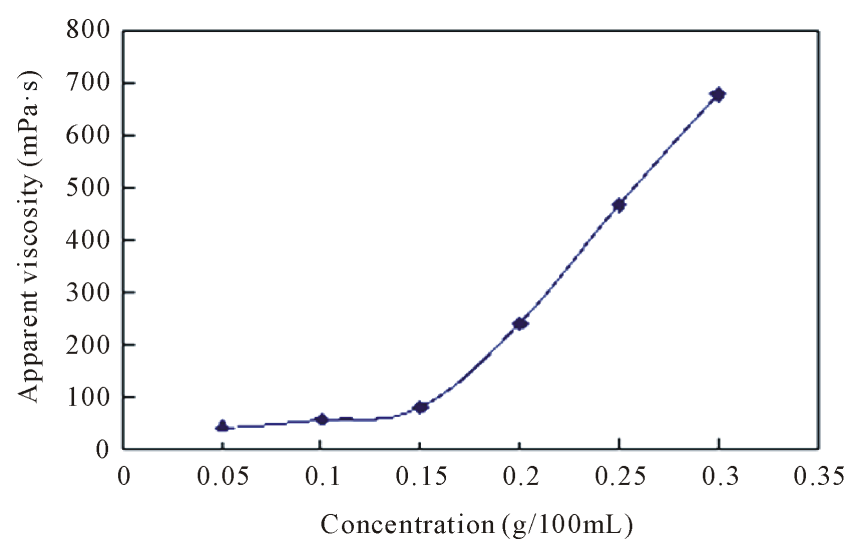

Figure 2. Hydrophobic associating concentration effects on apparent viscosity.

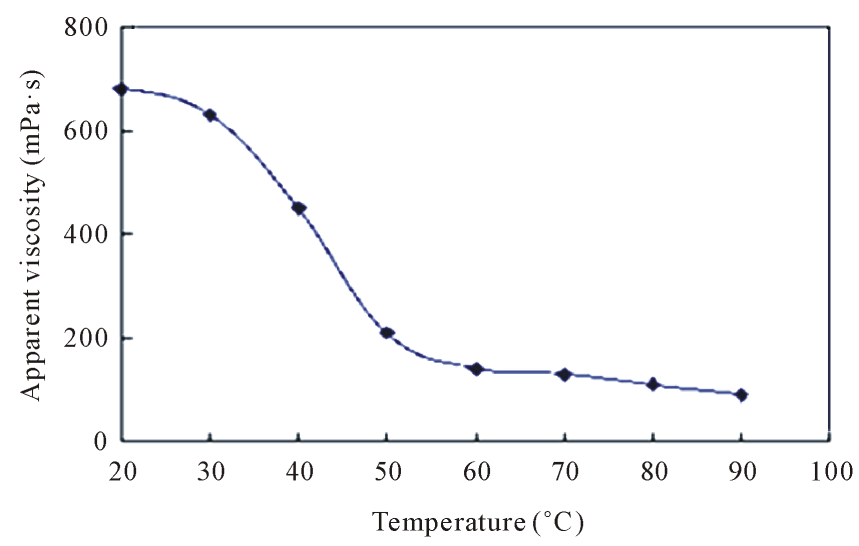

Figure 3. Temperature on the influence of the apparent viscosity of hydrophobic association. 


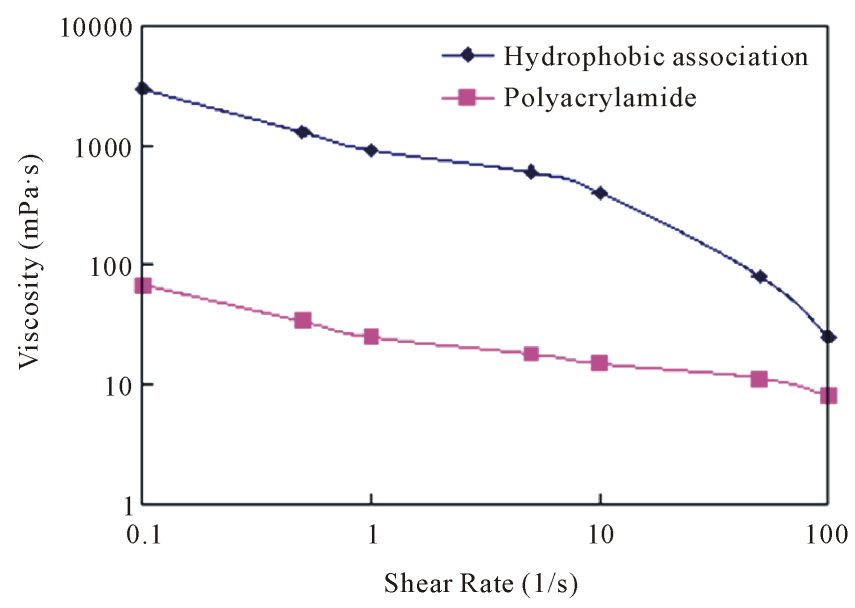

Figure 4. The influence of shear rate on viscosity of association.

main reason for decline in viscosity.

\subsection{Different Molecular Conformation of Polymer Solution Rheology}

Take the concentration is $2.50 \mathrm{~g} / \mathrm{L}$ of hydrophobic association solution and oilfield commonly used 18 million molecular weight polyacrylamide solution. Figure 4 shows both polymer shear thinning resistance, viscosity drop dramatically with the increase of shear rate. Under the same shear rate of hydrophobic association content solution viscosity is far higher than that of polyacrylamide solution viscosity.

\section{Conclusion}

Experiment shows that the hydrophobic association could form a special structure, which has higher viscosity and viscoelasticity. The complex fracturing fluid with hydrophobic association and surface active agent has temperature resistance and salt resistance. When the temperature is up to $90^{\circ} \mathrm{C}$, and the simulated formation water still has high viscosity, it can meet the requirements of fracturing with complete gum breaking, high discharge rate and less damage to the bottom.

\section{Acknowledgements}

The project is funded by the National Natural Science Foundation of the Study of Mechanism on Oil Soluble Resin-dual Cross-linked-gelatinous Water Shutoff Agent. Fund No.: 51374071.

\section{References}

[1] Bakeev, V.N., Yang, M.S., Zezin, A.B., et al. (1996) Structure and Properties of Poly Electrolyte-Surfactant Nonstoichiometric Complexes in Low-Polarity Solvents. Macromolecules, 29, 1320-1325. http://dx.doi.org/10.1021/ma9511311

[2] Thalberg, K., Lindman, B. and Karlstrom, G. (1991) Phase Behavior of Systems of Cationic Surfactant and Anionic Polyelectrolyte: Influence of Surfactant Hain Length and polyelectrolyte Molecular Weight. The Journal of Physical Chemistry, 95, 3370-3376. http://dx.doi.org/10.1021/j100161a073

[3] Thalberg, K., Lindman, B. and Karlstrom, G. (1991) Phase Behavior of a System of Cationic Surfactant and Anionic Poly Electroloyte: The Effects of Salt. The Journal of Physical Chemistry, 95, 6004-6011. http://dx.doi.org/10.1021/j100168a053

[4] Philippova, O.E., Hourdet, D., Khokhlov, A.R., et al. (1996) Interaction of Hydrophobically Modified poly(acrylic acid)hydrogels with Ionic Surfactants. Macromolecules, 29, 2822-2830. http://dx.doi.org/10.1021/ma951006p

[5] Anghel, D.F., Saito, S., Iovescu, A., et al. (1998) Interaction between poly-(acrylic acid) and Nonionic Surfactants with the Same Poly but Different Hydrophobic Moieties. Langmuir, 14, 5342-5346. 
Scientific Research Publishing (SCIRP) is one of the largest Open Access journal publishers. It is currently publishing more than 200 open access, online, peer-reviewed journals covering a wide range of academic disciplines. SCIRP serves the worldwide academic communities and contributes to the progress and application of science with its publication.

Other selected journals from SCIRP are listed as below. Submit your manuscript to us via either submit@scirp.org or Online Submission Portal.
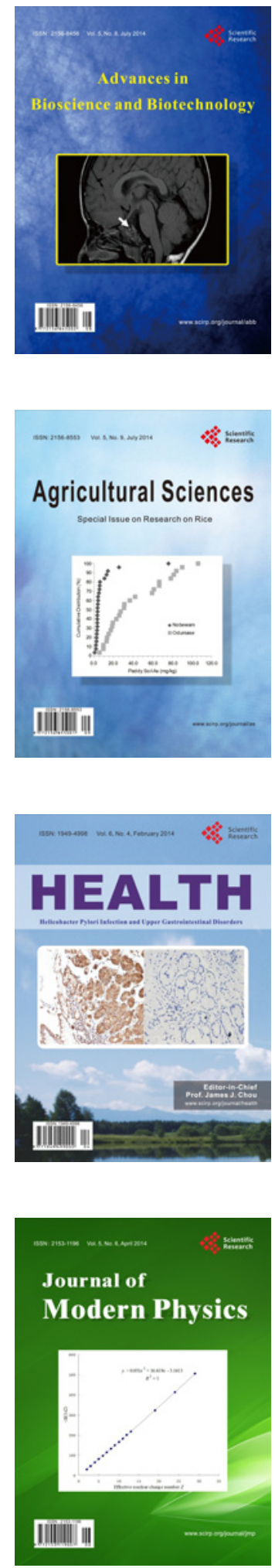
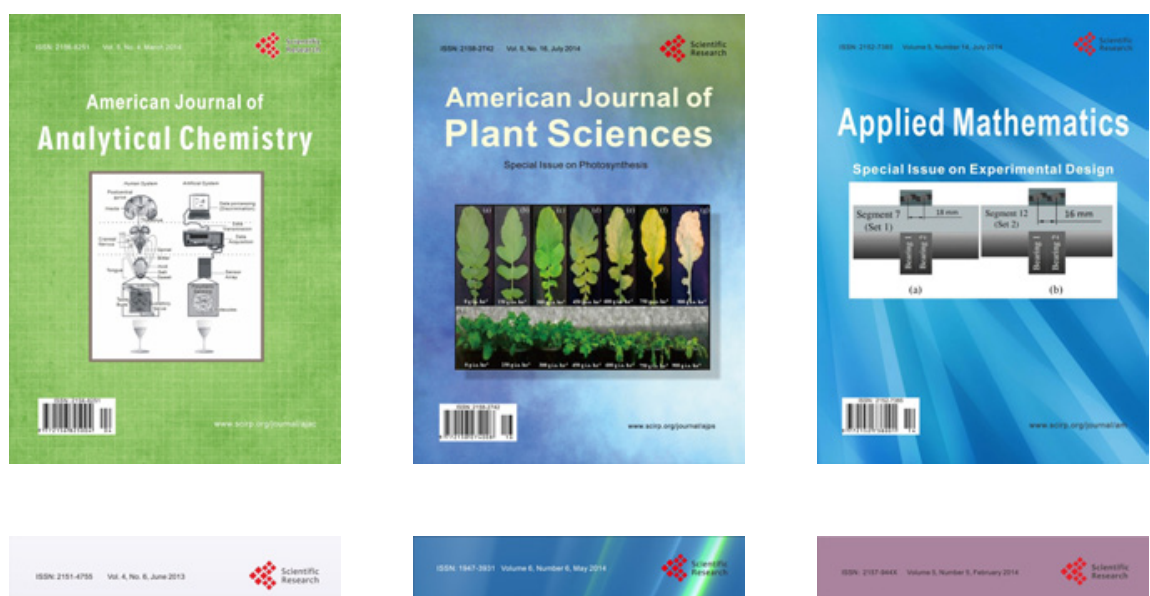

Creative Education
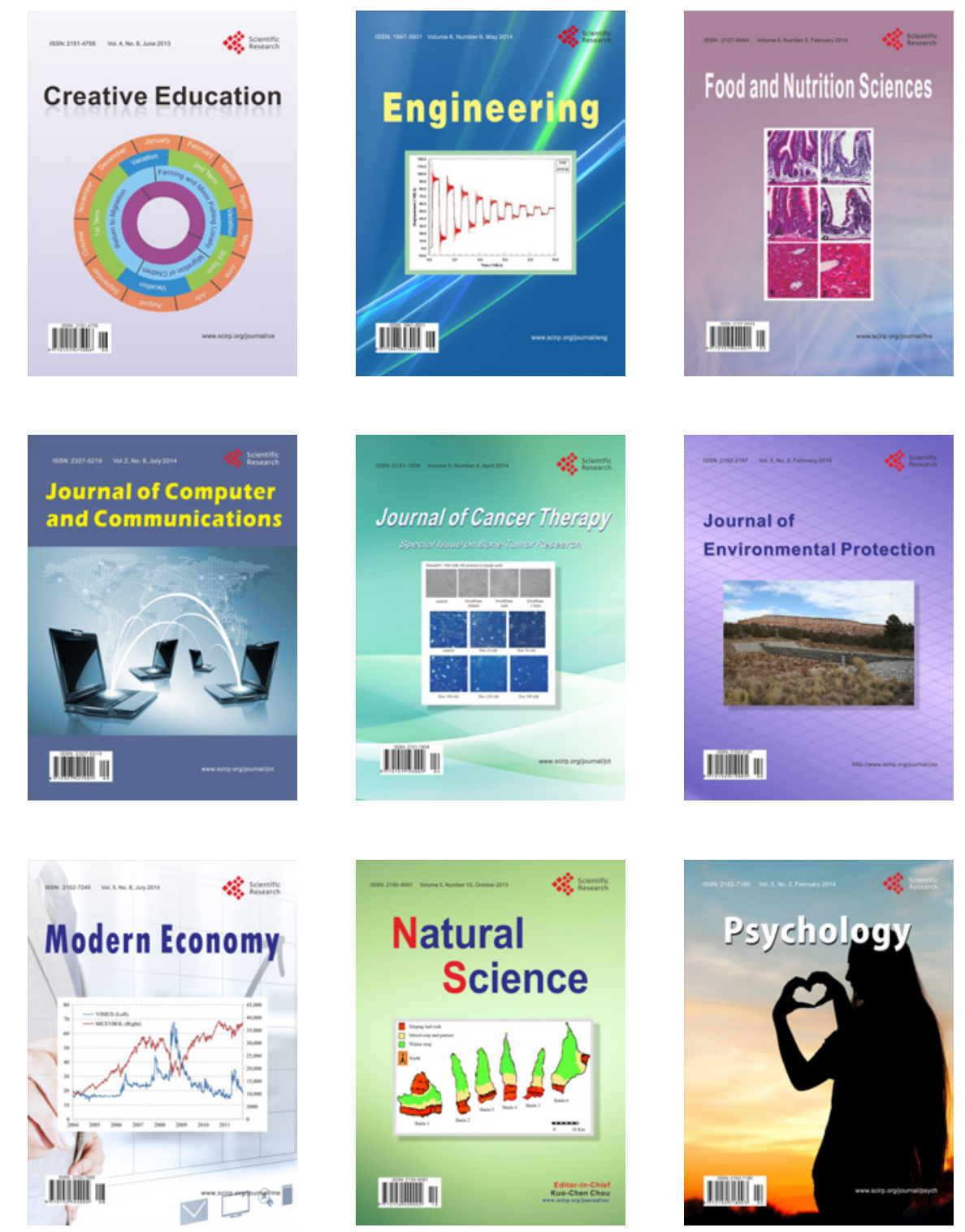